\title{
RENAL AFFECTION AND SOME OXIDATIVE STRESS BIOMARKERS AMONG WORKERS EXPOSED
}

\section{TO SILICA DUST}

\author{
By \\ Fahmy, F.C. ${ }^{1}$, Abdel-Hamid, M.A. ${ }^{2}$, Abbas, F. ${ }^{1}$ and El-Gazzar, R.M. ${ }^{1}$ \\ ${ }^{1}$ Department of Occupational Health, High Institute of Public Health, Alexandria University \\ ${ }^{2}$ Department of Community, Environmental and Occupational Medicine,
}

Faculty of Medicine, Ain-Shams University

\begin{abstract}
:
Occupational exposure to silica dust especially its crystalline form is primarily related to silicosis. Other possible silica related diseases such as renal disorders are still underestimated. The objective of the present work was to study the posibility of renal affection; as well as, alterations of some oxidative stress and autoimmune biomarkers among workers exposed to silica dust with and without silicosis. Methods: A crosssectional study was conducted in one of the factories for refractories in Alexandria, Egypt. The sample was divided into 3 groups; exposed silicotic, exposed non-silicotic and non-exposed group. Each group consisted of 27 workers. According to a parallel environmental survey, exposure levels to respirable free silica in the three departments of the plant namely; milling, mixing and production were identified. All participants were subjected to a predesigned questionnaire, clinical examination, anthropometric measurements, a standard chest radiological examination and laboratory investigations namely urinary albumin, antioxidants activity (SOD) and glutathione S-transferase and serum anti-neutrophil cytoplasmic antibodies (ANCA).
\end{abstract}

Results: The study shows that about 52\% of the exposed workers with silicosis, $40.7 \%$ of exposed workers with no silicosis, and $7.4 \%$ of non-exposed workers had microalbuminurea. There was no statistically significant difference in urinary albumin levels between exposed silicotic and exposed non-silicotic workers. There was a statistically significant difference between the 3 groups regarding their antioxidants levels. Qualitative ANCA testing results were negative for all workers. Multiple 
regression analysis showed that the most important predictors of urinary albumin level were age, duration of exposure, level of exposure to silica, SOD and Glutathione S-transferase (Adjusted $R \square=0.387$ ); the most important predictors of SOD level were duration of exposure and level of exposure to silica (Adjusted $R \square=0.692$ ); and that the most important predictors of Glutathione S-transferase level were duration of exposure, level of exposure to silica and pneumoconiotic score (Adjusted $\mathrm{R} \square=0.657$ ).

Conclusion, long-term exposure to silica dust may induce oxidative stress which plays a possible role in the renal affection among exposed workers with or without silicosis.

Key words: Silica exposure, Renal affection, Microalbuminurea, Oxidative stress, Superoxide dismutase, Glutathione.

Abbreviations: ANCA, anti-neutrophil cytoplasmic antibody; BMI, body mass index; SI, smoking index; SOD, superoxide dismutase

\section{Introduction}

Occupational exposure to silica dust especially its crystalline form is primarily related to silicosis which is a wellknown fibrogenic lung disease. Silicosis mortality trends are markedly decreasing in industrialized countries; meanwhile, the situation is worse in developing countries (Bang, et al., 2008; Rosenman, et al., 2009). Nevertheless, other possible silica related diseases such as renal disorders are still underestimated. Steenland, et al., (2001) reported an increase in pathological changes of renal functions from microalbuminuria to end-stage kidney disease among silica exposed workers. On the other hand, no renal mortality cases were reported among silicotics in two American State-based surveillance systems (Rosenman, et al., 1995). Gregorini, et al., (1997) claimed that renal affection was an infrequent observation in association with silica exposure and was an autoimmune anti-neutrophil cytoplasmic antibody (ANCA) related mechanism.

It has been stated that microalbuminuria is a sensitive marker for early renal involvement in chronic diseases (Foster, et al., 2007). However, markers of possible renal affection were quite variable in the different health surveillance studies of silica exposed workers. Moreover, the association between silicosis and renal disorders was inconsistent finding (NIOSH, 2002).

Although the exact mechanisms involved in the development of silicarelated diseases have not been fully explained, one assumption was the state of biological oxidative stress reported in relation to silica dust exposure (Shen, et al., 2001). Generating reactive oxygen species was raised recently as a possible mechanism inducing oxidative stress that 
leads to progression of many autoimmune disorders, renal diseases and silicosis (Kalpakcioglu, and Senel, 2008 \& Rimal, et al., 2005). The superoxide dismutases (SOD) are a family of antioxidant enzymes that play a pivotal role protecting tissues by scavenging superoxide anions (Goa, et al., 2008). Superoxide dismutase shows variable responses, especially in case of inflammation or ischemic injury of parenchymal organs including kidney (McCord, 2008). Also, glutathione is a key antioxidant multi-component mechanism that plays an important role in modifying and controlling inflammation with preventive promises (Perricon, et al., 2009). An early research showed that glutathione in red blood cells was increased in silicotic patients (Borm, et al., 1986). Other researches showed that glutathione content was decreased in silicotic lung tissues in rats in the early stage (Ghio, et al., 1994) and increased in the late stage (Yamano, et al., 1995). However, the situation of oxidative stress in silica induced disease especially in relation to possible renal affection is still unclear. Gulumian, et al., (2006) emphasized the importance for the development of biomarkers to improve the process of risk assessment and to identify the initiation of pathological changes prior to the development of the occupational disease especially in relation to silica exposure.

Exposure to silica dust represents one of the common occupational health problems in many industries. About 40,000 workers are estimated to be exposed to silica dust in Egypt (General Health Insurance Organization, 2001).

The aim of the present work was to study the possibility of renal affection; as well as, alterations of some oxidative stress and autoimmune biomarkers among workers exposed to silica dust with and without silicosis.

\section{Subjects and methods}

Study design and setting:

A cross-sectional study was conducted in one of the factories for refractories in Alexandria. The exposure departments included milling, mixing and production. The administrative sector represented the non-exposed group in the study.

Data collection:

Confidentiality was ensured. Ethical considerations were applied after getting permissions from concerned authorities; detailed explanation of the study procedures was done after which all participants gave a written consent to participate in the study. 
Environmental data:

Records of a parallel environmental survey were reviewed, showing exposure levels to respirable free silica of $0.1,0.15$ and $0.09 \mathrm{mg} / \mathrm{m}^{3}$ in the three departments of the plant namely; milling, mixing and production respectively.

Study population:

Using an assumed prevalence of renal affection among silica exposed and non-exposed workers of $40 \%$ and $5 \%$ respectively (Rosenman et al. 2009 and Rosenman et al. 2000) with $95 \%$ confidence and $80 \%$ power, the minimum required sample size is 27 for each group (Epi Info, 2004).

Workers free from chronic diseases causing microalbuminurea such as hypertension, diabetes mellitus, renal diseases and urinary disorders were only included in the study to avoid bias of the results (Stevens, et al., 2006).

All male exposed workers $(\mathrm{n}=337)$ were classified into silicotic and nonsilicotic workers, through screening by chest radiologic microfilms for all male workers in the three exposure departments. Cases that showed suspected opacities indicative of any stage of silicosis were re-examined by a standard chest X-ray film $(n=41)$. Silicotic cases were diagnosed according to the ILO International Classification of Radiographs of the Pneumoconioses (International Labor organization, 2000). All films were examined independently and compared to standard radiographs by two experienced occupational health physicians and a radiology consultant in Alexandria Health Insurance. In case of disagreement about any case they were asked to give a final diagnosis.

A group of exposed silicotic cases fulfilling the inclusion criteria were randomly selected for the study $(n=27)$. Two other groups each of 27 workers were selected by systematic random technique from exposed workers with no silicosis and the non-exposed administrative department after insuring that they met the inclusion criteria. In case that one subject was excluded the next name was considered. The three groups were matched for sociodemographic characteristics. Accordingly, the sample was divided into 3 groups:

- Exposed silicotic group $(\mathrm{n}=27)$

- Exposed non-silicotic group $(\mathrm{n}=$ 27)

- Non-exposed group $(\mathrm{n}=27)$

Study methods:

All participants were subjected to the following: 
- A predesigned questionnaire about personal, medical and smoking histories, as well as occupational data including duration of employment and department of work (NIOSH, 2002).

- Clinical examination, general and systematic, with particular emphasis on respiratory, cardiovascular and renal systems to ensure compliance of the study population with the inclusion criteria (Stevens, et al., 2006).

- Measurements of height $(\mathrm{cm})$ and weight $(\mathrm{kg})$ by the standard methods. Body mass index was calculated (BMI $\mathrm{kg} / \mathrm{m} 2$ ) and classified according to the WHO, (2006).

- Standard chest radiological examination to confirm cases of silicosis (as mentioned before); as well as, to exclude any chronic chest condition among exposed non-silicotic and non-exposed groups according to the ILO (International Labor Organization, 2000).

- Laboratory investigations:

a) Urinary albumin measurement: The second morning spot urine sample was collected for determining the ratio of urinary albumin to creatinine (mg/ gm urinary creatinine) by the DCA 2000 analyser (Siemens Healthcare Diagnostics Inc. Deerfield, US). Microalbuminurea was diagnosed at the level of $\geq 30 \mathrm{mg} / \mathrm{gm}$ creatinine (Foster, et al., 2007).

b) Antioxidants activity (SOD and glutathione S-transferase): A venous blood sample was obtained by veinpuncture and was left to clot. Serum was separated to measure some biologic markers related to oxidative stress. Superoxide dismutase (SOD) activity and glutathione S-transferase were measured according to methods of Paoletti, et al., (1986) in $\mathrm{u} / \mathrm{mg}$ protein and Habig, et al., (1974) in u/l respectively.

c) Serum anti-neutrophil cytoplasmic antibodies: c-ANCA and $\mathrm{p}$ status were also determined by enzyme linked immunosorbent assay (ELISA), using commercial antibody panel by MedTek Kits (Holle, et al., 2005).

Statistical analysis:

Data entry and analysis were performed using PASW program version 18. Based on environmental measurements, the level of exposure was ranked $0,1 \& 2$ for the 
non-exposed, mild exposure (milling and production) and high exposure (mixing) groups respectively. In order to quantify the severity of ILO classification of silicosis, a pneumoconiotic score was calculated considering the sum of lung zones affected both in right and left lungs multiplied by size of opacity ( $\mathrm{p}=1 ; \mathrm{q}=2 ; \mathrm{r}=3$ ) and multiplied by category of profusion (category 1 [mild] = 1/0,1/1, 1/2; category 2 [moderate] $=2 / 1,2 / 2,2 / 3$; category 3 [severe] $=3 / 2,3 / 3,3 /+)$ (Rosenman, et al., 2009).

The data collected were analyzed by descriptive as well as inferential analysis. Frequency distribution tables, descriptive analysis (mean and standard deviation) as well as descriptive bar charts were used. As for the inferential part, chi-squared test, student t-test, one way ANOVA test and Pearson correlation analysis were applied. Based on the results of the univariate analysis, multiple linear regression analysis was performed to select all the independent variables that are believed to have significant effect on the quantitative dependent variable. Backward method was used. The estimated model provides the direct effect of each independent variable on the dependent variable. Such effect is the net effect after fixing all effects of other independent variables included in the model. The multiple linear regression models look for the best model that minimizes the vertical distance between the fitted line and the observed value of the dependent variable. The significance level was set at $\mathrm{P}<0.05$.

\section{Results}

Eighty one workers were included in the study. Fifty four of them were exposed to silica dust during their work and 27 workers were not exposed. About $57 \%$ of the workers participating in the study were under 40 years old with a mean age of $39.31 \pm 6.67$ years. More than half of them $(55.6 \%)$ were non-smokers. The duration of employment in the current jobs was between 10 and 20 years in most workers. Twenty workers were working in the milling department, 20 in the mixing department, 14 in the production department, and 27 workers in the administration department. There were no statistically significant differences for the age, body mass index (BMI), smoking habit and duration of work of the participating workers according to their exposure to silica dust and the presence of silicosis (table 1).

Of the 27 silicotic patients, 16 had an ILO profusion of small opacities $1 / 1,7$ had an ILO profusion of $1 / 2$, and 4 had an ILO profusion of $2 / 2$. The extent of lung opacity, 
in both right and left lung zones, included 2 lung zones in 6 workers $(22.2 \%), 3$ lung zones in 9 workers (33.3\%), 4 lung zones in 7 workers $(25.9 \%)$, and 5 lung zones in 5 workers $(18.5 \%)$. All the 27 patients had small regular opacities, the size of the opacity in 18 of them was up to about $1.5 \mathrm{~mm}$ 'p' and it was exceeding about $1.5 \mathrm{~mm}$ and up to about $3 \mathrm{~mm}$ ' $\mathrm{q}$ ' in 9 of them according to the ILO International Classification of Radiographs of the Pneumoconioses. The mean pneumoconiotic score of silicotic workers was $5.82 \pm 4.74$ (table 2).

Results of the study showed that about $52 \%$ of the exposed workers with silicosis, $40.7 \%$ of the exposed workers with no silicosis, and $7.4 \%$ of the non-exposed workers had microalbuminurea. Moreover, one way ANOVA test showed that there was no statistically significant difference in urinary albumin levels between exposed silicotic and exposed non-silicotic workers. It also showed that there was a statistically significant difference in urinary albumin levels between the non-exposed group and the exposed groups (either silicotic or non-silicotic). The antioxidants levels were found to be statistically significantly different between the 3 groups of the participating workers according to exposure to silica dust and the presence of silicosis. The levels of SOD ( $\mathrm{u} / \mathrm{mg}$ protein) showed that exposed workers with silicosis had the minimum SOD levels $(4.427 \pm 0.427)$ followed by exposed workers with no silicosis (4.743 \pm 0.562$)$. Meanwhile, nonexposed workers had the maximum SOD level (6.472 \pm 0.517$)$. Also, the glutathione S-transferase levels ( $\mathrm{u} / \mathrm{l})$ among exposed workers with or without silicosis were found to be significantly lower $(4.395 \pm 0.441$ and $4.560 \pm 0.447$ respectively) than nonexposed workers (5.886 \pm 0.273$)$ (table 3$)$.

The possible mechanism of renal affection among workers exposed to silica dust was assessed. Results showed that the antioxidants levels were significantly lower in presence of positive microalbuminurea than among negative cases (SOD: $4.27 \pm 0.29$ vs $4.86 \pm 0.52 \mathrm{u} / \mathrm{mg}$ protein; $\mathrm{t}=5.06 \mathrm{p}<0.001$ \& Glutathione S-transferase: $4.26 \pm 0.29$ vs $4.66 \pm 0.48 \mathrm{u} / \mathrm{l} ; \mathrm{t}=3.65 \mathrm{p}<0.001)$. On the other hand, qualitative ANCA testing results were negative for all workers regardless of urinary albumin levels.

Pearson correlation analysis revealed that:

- Urinary albumin level was positively correlated with age, duration of exposure and level of exposure.

- Urinary albumin level was negatively correlated with SOD and glutathione s-transferase. 
- Urinary albumin level was not correlated with smoking index, BMI and pneumoconiotic score

- The SOD level was negatively correlated with duration of exposure, level of exposure and pneumoconiotic score

- The SOD level was not correlated with age, smoking index and BMI

- Glutathione S-transferase level was negatively correlated with duration of exposure, level of exposure and pneumoconiotic score

- Glutathione S-transferase level was not correlated with age, smoking index and BMI (table 4).

According to the results of Pearson correlation, proposed models for the independent factors affecting urinary albumin levels, SOD and Glutathione S-transferase levels were put. Then multiple linear regressions (backward method) were applied on the proposed models to find the significant factors affecting these dependent variables.
The most important predictors of urinary albumin level, in multiple regression analysis, were age, duration of exposure, level of exposure, SOD and Glutathione S-transferase. This model explained about $38 \%$ of the variation on this dependent variable (Adjusted R曰0.387).

The regression was run two (2) times using backward method for the SOD level. It showed that the most important predictors of this dependent variable were duration of exposure and level of exposure. On the other hand, the pneumoconiotic score was excluded from the equation. This model explained about $69 \%$ of the variation on SOD level (Adjusted R曰0.692).

The multiple regression analysis of glutathione S-transferase level showed that the most important predictors were duration of exposure, level of exposure and pneumoconiotic score. This model explained about $65 \%$ of the variation on this dependent variable (Adjusted R $\boxminus 0.657$ ). (table 5) 
Table 1: Characteristics of the studied sample according to exposure to silica dust and the presence of silicosis $(\mathrm{N}=81)$.

\begin{tabular}{|c|c|c|c|c|c|c|c|c|c|c|}
\hline & \multicolumn{2}{|c|}{$\begin{array}{l}\text { Exposed } \\
\text { silicotic } \\
(\mathbf{n}=27)\end{array}$} & \multicolumn{2}{|c|}{$\begin{array}{c}\text { Exposed } \\
\text { non- silicotic } \\
(\mathbf{n}=27)\end{array}$} & \multicolumn{2}{|c|}{$\begin{array}{c}\text { Non } \\
\text { exposed } \\
(\mathbf{n}=27)\end{array}$} & \multicolumn{2}{|c|}{$\begin{array}{c}\text { Total } \\
(\mathrm{N}=\mathbf{8 1})\end{array}$} & \multirow[t]{2}{*}{$\mathbf{X} \square$} & \multirow[t]{2}{*}{ p-value } \\
\hline & $\mathbf{n}$ & $\%$ & $\mathbf{n}$ & $\%$ & $\mathbf{N}$ & $\%$ & $\mathbf{N}$ & $\%$ & & \\
\hline \multicolumn{11}{|l|}{ Age (in years) } \\
\hline$<40$ & 15 & 55.6 & 16 & 59.3 & 15 & 55.6 & 46 & 56.8 & \multirow[t]{2}{*}{0.101} & \multirow[t]{2}{*}{0.951} \\
\hline$\geq 40$ & 12 & 44.4 & 11 & 40.7 & 12 & 44.4 & 35 & 43.2 & & \\
\hline Mean \pm SD & \multicolumn{2}{|c|}{$39.33 \pm 7.071$} & \multicolumn{2}{|c|}{$39.19 \pm 6.505$} & \multicolumn{2}{|c|}{$39.41 \pm 6.664$} & \multicolumn{2}{|c|}{$39.31 \pm 6.67$} & & \\
\hline \multicolumn{11}{|l|}{ Body Mass Index (kg/m²) } \\
\hline normal & 9 & 40.9 & 7 & 31.8 & 6 & 27.3 & 22 & 27.2 & \multirow{3}{*}{1.179} & \multirow{3}{*}{.882} \\
\hline overweight & 13 & 28.9 & 16 & 35.6 & 16 & 35.6 & 45 & 55.6 & & \\
\hline obese & 5 & 35.7 & 4 & 28.6 & 5 & 35.7 & 14 & 17.3 & & \\
\hline Mean \pm SD & 26.75 & 3.04 & 26.9 & 2.53 & 27.47 & .12 & $27.0^{\circ}$ & 2.89 & & \\
\hline \multicolumn{11}{|l|}{ Smoking } \\
\hline Yes & 11 & 40.7 & 12 & 44.4 & 13 & 48.1 & 45 & 55.6 & \multirow[t]{2}{*}{0.300} & \multirow[t]{2}{*}{0.861} \\
\hline No & 16 & 59.3 & 15 & 55.6 & 14 & 51.9 & 36 & 44.4 & & \\
\hline \multicolumn{11}{|l|}{$\begin{array}{l}\text { Smoking Index }(\mathbf{S I}) \\
\text { (Daily cigarettes*years) }\end{array}$} \\
\hline <200 (light) & 0 & 0 & 0 & 0 & 0 & 0 & 0 & 0 & \multirow{3}{*}{0.235} & \multirow{3}{*}{0.889} \\
\hline $200-<400$ (moderate) & 4 & 26.7 & 5 & 33.3 & 6 & 40 & 15 & 41.7 & & \\
\hline$\geq 400$ (heavy) & 7 & 33.3 & 7 & 33.3 & 7 & 33.3 & 21 & 58.3 & & \\
\hline Mean \pm SD & \multicolumn{2}{|c|}{$400 \pm 148.32$} & \multicolumn{2}{|c|}{$408.33 \pm 178.16$} & \multicolumn{2}{|c|}{$407.69 \pm 210.01$} & \multicolumn{2}{|c|}{$405.56 \pm 177.19$} & & \\
\hline \multirow{2}{*}{\multicolumn{11}{|c|}{$\begin{array}{l}\text { Duration of work } \\
\text { (in years) }\end{array}$}} \\
\hline & & & & & & & & & & \\
\hline$<10$ & 5 & 18.5 & 4 & 14.8 & 3 & 11.1 & 12 & 14.8 & \multirow{3}{*}{1.304} & \multirow{3}{*}{0.861} \\
\hline $10-$ & 15 & 55.6 & 17 & 63.0 & 19 & 70.4 & 51 & 63.0 & & \\
\hline$\geq 20$ & 7 & 25.9 & 6 & 22.2 & 5 & 18.5 & 18 & 22.2 & & \\
\hline Mean \pm SD & 14.3 & \pm 5.173 & 14.2 & \pm 5.096 & 14.2 & 4.864 & & \pm 4.98 & & \\
\hline Department & & & & & & & & & & \\
\hline milling & 10 & 37.0 & 10 & 37.0 & - & - & 20 & 24.7 & & \\
\hline mixing & 10 & 37.0 & 10 & 37.0 & - & - & 20 & 24.7 & 81.00 & $<0.001$ \\
\hline production & 7 & 25.9 & 7 & 25.9 & - & - & 14 & 17.3 & & \\
\hline administration & - & - & - & - & 27 & 100 & 27 & 33.3 & & \\
\hline
\end{tabular}


Table 2: Chest radiography of silicotic workers and their pneumoconiotic score $(n=27)$.

\begin{tabular}{|ccc|}
\hline & N & $\%$ \\
\hline Profusion of lung opacity & 16 & 59.3 \\
1/1 (mild) & 7 & 25.9 \\
1/2 (mild/ moderate) & 4 & 14.8 \\
2/2 (moderate) & & \\
\hline Extent of opacity (Right and Left lung zones) & 6 & 22.2 \\
2 lung zones & 9 & 33.3 \\
3 lung zones & 7 & 25.9 \\
4 lung zones & 5 & 18.5 \\
5 lung zones & & \\
\hline Size of lung opacity * & 18 & 66.7 \\
p & 9 & 33.3 \\
\hline Pneumoconiotic score & & \\
Mean \pm SD & & $5.82 \pm 4.74$ \\
\hline
\end{tabular}

$* \mathrm{P}=$ diameter up to about $1.5 \mathrm{~mm}$

$\mathrm{q}=$ diameter exceeding about $1.5 \mathrm{~mm}$ and up to about $3 \mathrm{~mm}$ 


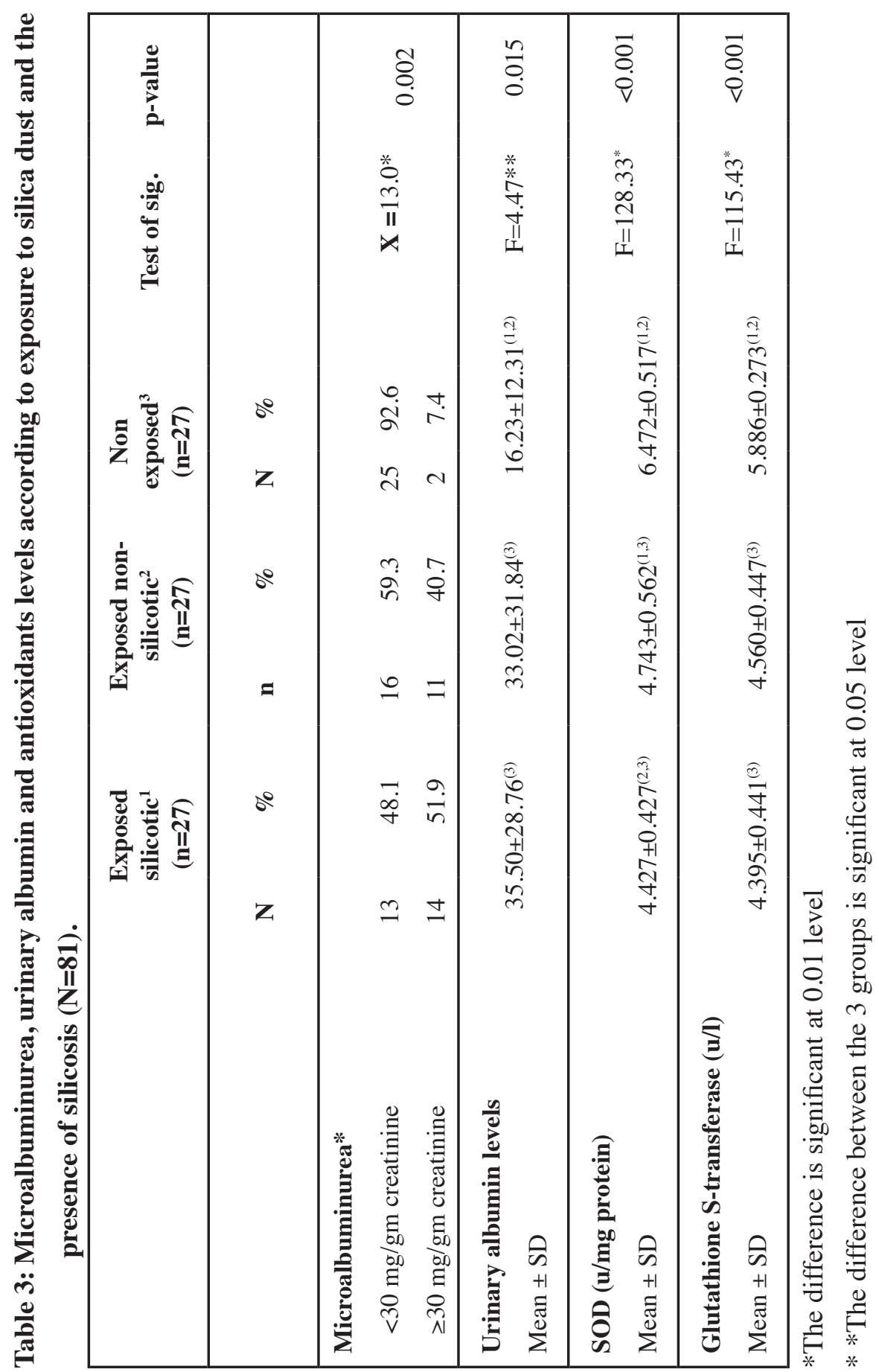


Table 4: Correlation between urinary albumin levels, SOD and Glutathione S-transferase and different independent factors.

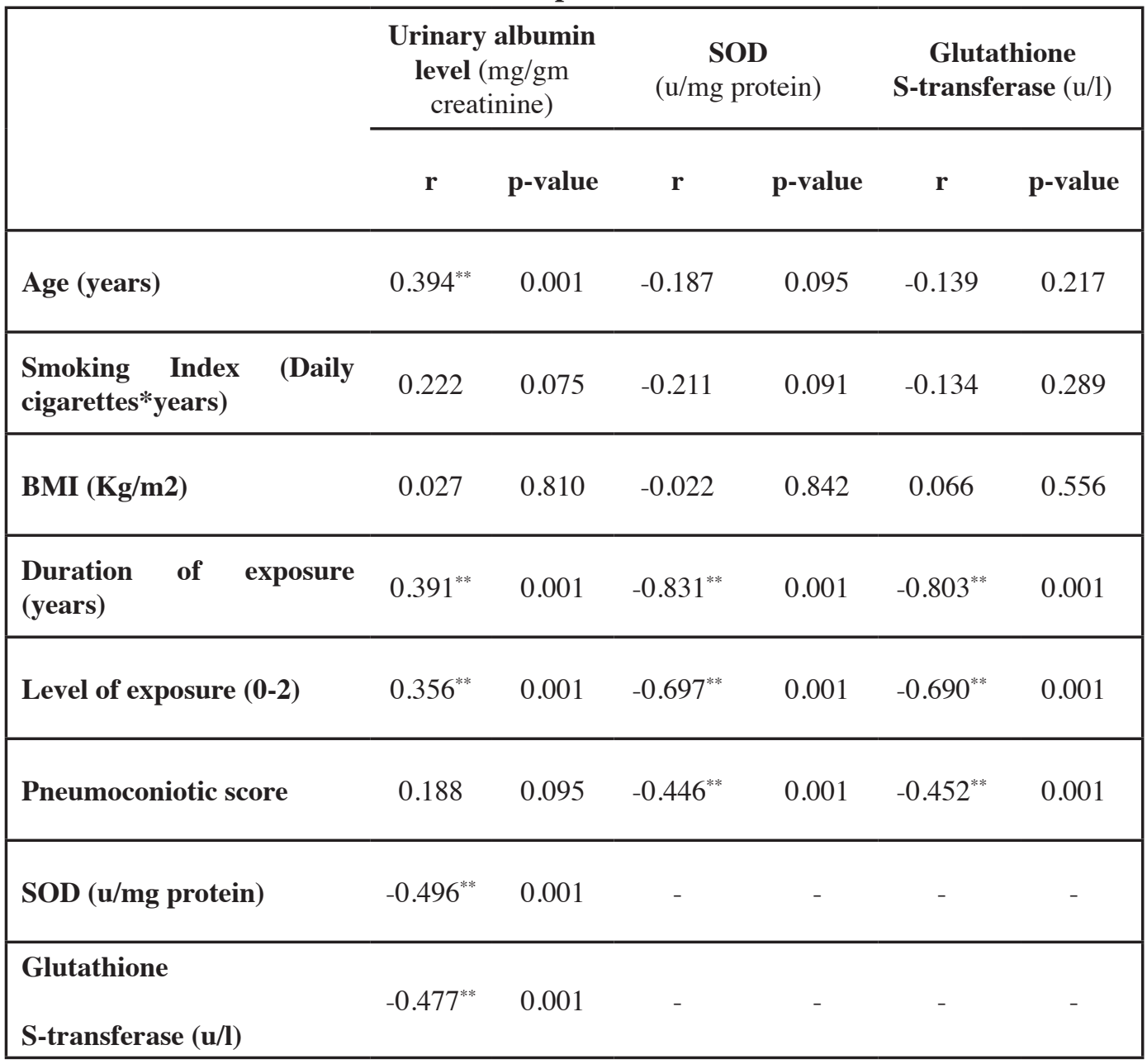

**. Correlation is significant at the 0.01 level (2-tailed). 


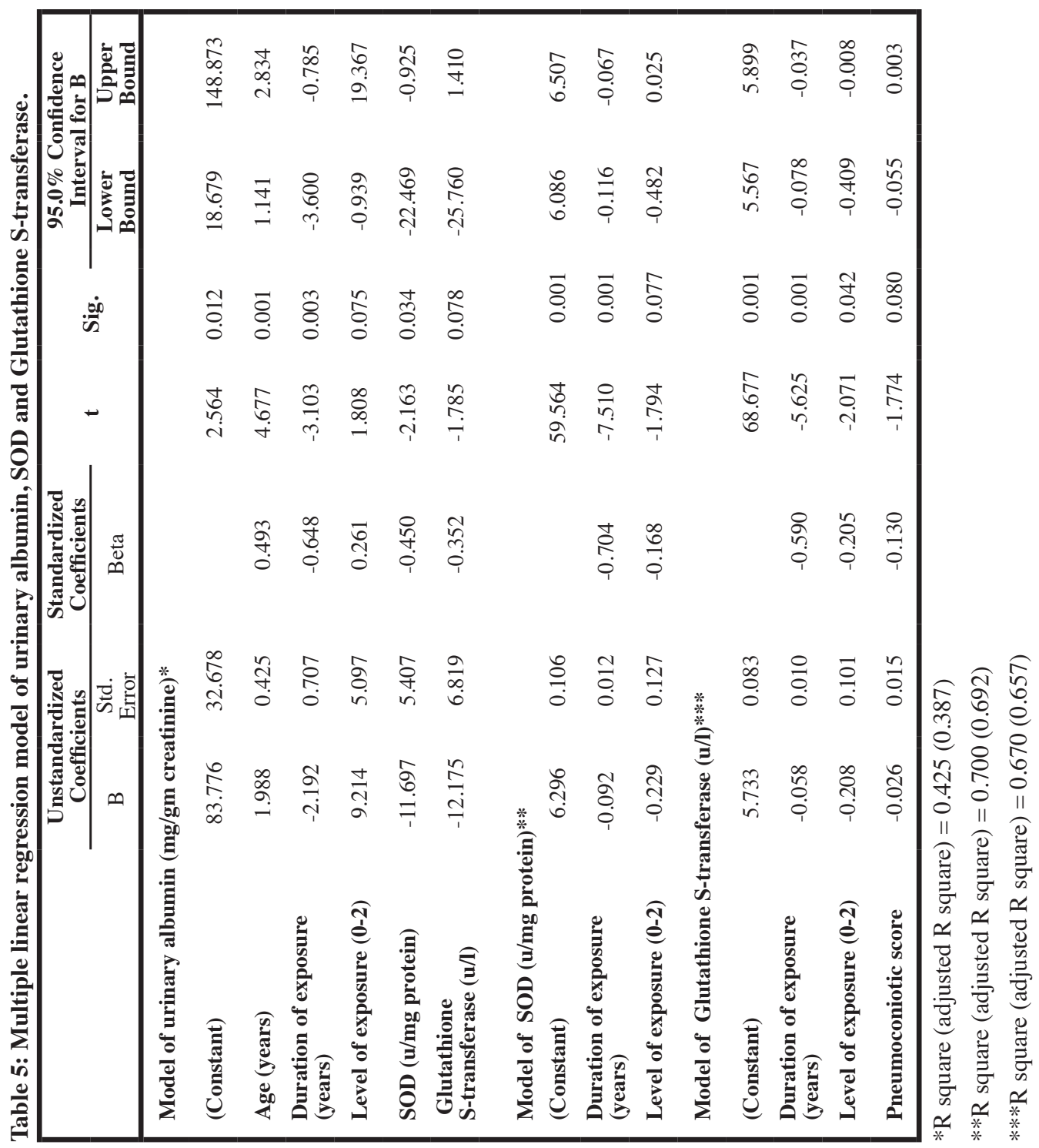




\section{Discussion}

The exposure level to silica dust exceeded the permissible level of exposure for respirable quartz $\left(0.05 \mathrm{mg} / \mathrm{m}^{3}\right.$, Egyptian Ministry of Trade and Industry, Law 12 Decision 211/2003). The participating groups in the present study were homogeneous and were matched by age, smoking habit and duration of employment.

Twenty seven male workers were diagnosed as having silicosis on the basis of their occupational history and the ILO International Classification of Radiographs of Pneumoconioses (International Labor Organization, 2000). It has been stated that the pneumoconiotic response of a worker to silica dust exposure is influenced to a great extent by susceptibility, meanwhile; the extent of silicosis is determined mainly by respirable silica concentration and duration of exposure (NIOSH, 2002). Therefore, the pattern of silicosis reported in this study, in workers with about 14 years mean duration of exposure, reflected a significant silica dust exposure dose.

Studying the relation between exposure to silica dust and urinary albumin level revealed that microalbuminurea, which is an indicator of early renal disease, was significantly more prevalent among workers exposed to silica dust than non-exposed workers. This finding is in agreement with the results of $\mathrm{Ng}$, et al., (1992) who were the first to undergo a controlled study to investigate early glomerular and tubular changes among silica exposed workers. They found a significantly higher urinary excretion of albumin and $\alpha_{1}$ microglobulin among them compared to the referents and they concluded that prolonged exposure to silica is associated with chronic irreversible nephrotoxicity in exposed workers. Also, it confirms the previous epidemiologic studies that found an association between kidney disease and exposure to silica (Rapiti, et al., 1999; Steenland, et al., 2001). The present study showed also that there was no statistically significant difference in urinary albumin levels between exposed workers suffering from silicosis and exposed workers not suffering from silicosis. This indicates that microalbuminurea, and thus renal disease, may occur in workers exposed to silica dust irrespective to the presence of silicosis. This finding confirms the results of Rapiti, et al., (1999) who provided further evidence that exposure to silica dust among ceramic workers was associated with nephrotoxic effects whether they were silicotic or not. Similarly, in a previous Egyptian research, 
the renal function parameters were found to be significantly higher in exposed groups (both silicotic and non-silicotic) than the non-exposed group (EL-Safty, et al., 2003). In addition, in the present study the duration of exposure to silica kept its significant effect on the urinary albumin level after adjustment for other confounders. This means that prolonged exposure to silica dust may lead to renal disease. Accordingly, an occupational history for exposure to silica must be obtained for all renal patients as stated by previous researchers (Stratta, et al., 2001). On the other hand, the present results contradict with the results of Rosenman, et al., (2000) who concluded that chronic kidney disease should be considered as a complication of silicosis.

Epidemiologic data are conflicting on the mechanism by which silica dust causes kidney disease and are compatible with silica being able to cause kidney disease by both an autoimmune and direct nephrotoxic effects (Rosenman, et al., 1995). Gregorini, et al., (1997) claimed that an autoimmune ANCA related mechanism may be responsible for end-stage renal disease among silica exposed workers. In the present study, quantitative ANCA testing revealed negative results for all workers regardless of urinary albumin level. This is going with the findings of Rimal, et al., (2005) in their review, who reported that the autoimmune markers were not consistently related to silica induced diseases and another mechanism may be involved such as oxidative stress.

Plasma antioxidant levels (SOD and glutathione S-transferase) were tested in the participating workers of the current study. Results showed that exposed silicotic workers had the lowest SOD levels followed by exposed non-silicotic workers and that non-exposed workers were found to have the highest SOD levels. Moreover, glutathione S-transferase levels among exposed workers with or without silicosis were significantly lower than nonexposed workers. Duration of exposure to silica dust was a significant determinant for both SOD and glutathione S-transferase in multivariate analysis of the present work. This may indicate that plasma antioxidants were consumed due to oxidative stress. This is going with the statement of Kinnula, and Carapo, 2003; who reported that most human studies suggest downregulation of SOD during fibrotic phases of parenchymal organ diseases. Also in support of the present study is the work of Zhang, et al., (1999), who demonstrated a decrease in a glutathione activity marker 
after silica exposure with a dose and timedependent manner. Also, Hamilton, et al., (2008) claimed that excessive amounts of free radicals are released from silicaactivated macrophages resulting in initial induction of cellular antioxidants as a defense mechanism followed by depletion of catalytic and scavenging antioxidants with cellular damage. On the contrary, a Chinese study showed that the SOD activity levels were higher in all silica exposed groups than controls, but not for glutathione (Zhang, et al., 2010). The discrepancy between results of the current study and the Chinese study may be attributed to differences in characterization of silica exposure, and duration of work in both studies. Moreover, lifestyle and ethnic differences may account for many changes in serum antioxidant levels (Nojima, et al., 2009).

In the present study, the antioxidant levels (SOD and glutathione S-transferase) were significantly lower in the presence of microalbuminurea, especially SOD which kept a significant effect in the multivariate analysis. Accordingly, this may indicate that the mechanism of kidney disease caused by prolonged exposure to silica dust is probably an oxidative stress. On the other hand, Day, (2008) reviewed the potential therapeutic effect of antioxidants in preventing progress of silica-induced disease, he reported that the newly developed antioxidants including SOD mimetics and glutathione like-scavengers antioxidants are effective in attenuating fibroproliferative responses to silica in animal models and some human studies.

\section{Conclusion and Recommendations}

In conclusion, long-term exposure to silica dust may lead to renal affection, as evidenced by microalbuminurea, regardless of the presence or absence of silicosis. Also, exposure to silica dust may induce oxidative stress in the form of changes in antioxidant biomarkers such as SOD and glutathione S-transferase. Oxidative stress is a possible mechanism for the renal affection among exposed workers with or without silicosis; rather than, an ANCA related autoimmune mechanism.

It is recommended to measure microalbuminurea in pre-placement and periodic medical examinations of silica exposed workers for early detection of renal affection. Workers with chronic kidney diseases should be added to the list of conditions prohibiting exposure to silica dust. Further studies are recommended to investigate the relationship between levels 
of SOD and glutathione S-transferase and their possible therapeutic effect as novel antioxidants in silica related health disorders. Emphasis should be given to environmental control of silica dust exposure and proper use of personal protective equipment.

\section{Acknowledgement is expressed} for Dr. Nagi Makari, the consultant of radiology in Alexandria Health Insurance for his participation in the interpretation of radiological examinations of this study.

\section{References}

1. Bang, K.M., Attfield, M.D., Wood, J.M. and Syamlal, G. (2008). "National trends in silicosis mortality in the United States, 1981-2004." Am. J. Ind. Med. 51(9): 633-9.

2. Borm, P.J., Bast, A., Wouters, E.F., Slangen, J.J., Swaen, G.M. and de Boorder, T. (1986). "Red blood cell anti-oxidant parameters in silicosis." Int. Arch. Occup. Environ. Health. 58: 235-44.

3. Day, B.J. (2008). "Antioxidants as potential therapeutics for lung fibrosis." Antioxid. Redox. Signal. 10(2): 355-70.

4. Egyptian Ministry of Trade and Industry Law 12 Decision 211/2003. (2008). "List of dangerous materials and its TLVs for high risk facilities." General Governmental Press. ed. 15: 509609. (in Arabic)

5. EL-Safty, I.A., Gadallah, M., Shouman, A.E. and Nessim, D.E. (2003). "Subclinical nephrotoxicity caused by smoking and occupational silica exposure among Egyptian industrial workers." Arch. Med. Res. 34(5): 415-21.

6. Epi Info, (2004). "Database and statistics software for public health professionals." CDC, Atlanta. Version 6.0.
7. Foster, M.C., Hwang, S.J., Larson, M.G., Parikh, N.I., Meigs, J.B., Vasan, R.S., wang, T.J., Levy, D. and Fox, C.S. (2007). "Crossclassification of microalbuminuria and reduced glomerular filtration rate: association between cardiovascular disease risk factors and clinical outcomes." Arch. Intern. Med. 167(13): 138692.

8. Gao, F., Kinnula, V.L., Myllamiemi, M. and Oury, T.D. (2008). "Extracellular superoxide dismutase in pulmonary fibrosis." Antioxid. Redox. signal. 10(2):343-54.

9. General Health Insurance Organization, (2001). "Permanent Committee for Planning, Supervision and Follow-up Implementation of Periodic Medical Examination." G.H.I.O. Cairo. (in Arabic)

10. Ghio, A.J., Jaskot, R.H., and Hatch, G.E. (1994). "Lung injury after silica instillation is associated with an accumulation of iron in rats." Am. J. Physiol. 267: L743-8.

11. Gregorini, G., Tira, P., Frizza, J., D'Haese, P.C., Elseviers, M.M., Nuyts, G.D., Maiorcar and DeBroe, ME. (1997). "ANCA-associated diseases and silica exposure." Clinic. Revi. Allerg. Immunol. 15: 21-40.

12. Gulumian, M., Borm, P.J.A., Vallyathan, V., Castranova, V., Donaldson, K., Nelson, G. and Murray, J. (2006). "Mechanically identified suitable biomarkers of exposure, effect, and susceptibility for silicosis and coal-worker's pneumoconiosis: A comprehensive review." J. Toxicol. Environ. Health, Part B. 9: 357-95.

13. Habig, W.H., Pabst, M.J. and Jajob, Y. (1974). "Glutathione S-transferases: The first enzymatic stepin mercapturic acid formation." J. Biol. Chem. 249: 7130-9.

14. Hamilton, R.F. Jr., Thakur, S.A. and Holian, A. (2008). "Silica binding and toxicity in alveolar macrophages." Free Radic. Biol. Med. 44: 1246-58. 
15. Holle, J.U., Hellmich, B., Backes, M., Gross, W.L. and Csernok, E. (2005). "Variations in performance characteristics of commercial enzyme immunoassay kits for detection of antineutrophil cytoplasmic antibodies: what is the optimal cut off?" Ann. Rheum. Dis. 64(12): 1773-9.

16. International Labor Organization, (2000). "Guidelines for the use of the ILO international classification of radiographs of pneumoconioses." Occupational Safety and Health Series No.22 I.L.O. Geneva, Switzerland.

17. Kalpakcioglu, B. and Senel, K. (2008). "The interrelation of glutathione reductase, catalase, glutathione peroxidase, superoxide dismutase, and glucose-6-phosphate in the pathogenesis of rheumatoid arthritis." Clin. Rheumatol. 27: 141-5.

18. Kinnula, V.L. and Crapo, J.D. (2003). "Superoxide dismutases in the lung and human lung diseases." Am. J. Respir. Crit. Care Med. 167(12): 1600-19.

19. McCord, J.M. (2008). "Superoxide dismutaze, lipid peroxidation, and bell-shaped dose response curves.” Dose-Response. 6: 223-38.

20. Ng, T.P., Ng, Y.L., Lee, H.S., Chia, K.S. and Ong, H.Y. (1992). "A study of silica nephrotoxicity in exposed silicotic and non-silicotic workers.” Br. J. Ind. Med. 49: 35-7.

21. NIOSH, (2002). "Hazard Review. Health Effects of Occupational Exposure to Respirable Crystalline Silica." Cincinnati Ohaio: DHHS (NIOSH) 129.

22. Nojima, M., Sakauchi, F., Mori, M., et al., (2009). "Relationship of serum superoxide dismutase activity and lifestyle in healthy Japanese adults." Asian. Pac. J. Cancer Prev. 10 Suppl: 37-40.

23. Paoletti, F., Aldinucci, D., Mocali, A. and Caarrini, A. (1986). "A sensitive spectrophotometric method for determination of superoxide dismutase activity." Ana. Biochem. 154: 536-41.
24. Perricone, C., Carolis, C.D. and Perricone, R. (2009). "Glutathione: A key player in autoimmunity." Autoimmunity Reviews. 8: 697-701.

25. Rapiti, E., Sperati, A., Miceli, M., Forastiere, F., Di Lallo, D., Cavariani, F., Goldsmith, D.F. and Perucci, C.A. (1999). "End stage renal disease among ceramic workers exposed to silica." Am. J. Ind. Med. 56(8): 559-61.

26. Rimal, B., Greenberg, A.K. and Rom, W.N. (2005). "Basic pathogenetic mechanisms in silicosis: current understanding." Current Opinion in Pulmonary Medicine. 11(2): 169-73.

27. Rosenman, K.D., Moore-Fuller, M. and Reilly, M.J. (2000). "Kidney Disease and Silicosis." Nephron. 85:19-9.

28. Rosenman, K.D., Reilly, M.J. and Kalinowski, D.J. (2009). "2007 Annual Report on Silicosis in Michigan." A Joint Report of the Mitchigan State University Department of Medicine and Department of Energy, Labour and Economic Growth. pp.: 1-10.

29. Rosenman, K.D., Stanbury, M.J. and Reilly, M.J. (1995). "Mortality among persons with silicosis reported to two state-based surveillance systems." Scand. J. work Environ. Health. 21(Suppl 2): 73-6.

30. Shen, H.M., Zhang, Z., Zhang, Q. and Ong, C. (2001). "Reactive oxygen species and cascade activation mediate silica-induced apoptosis in alveolar macrophages." Am. J. Physiol. 280: L10-L17.

31. Steenland, K., Sanderson, W. and Calvert, G.M. (2001). "Kidney disease and arthritis in a cohort study of workers exposed to silica." Epidemiology. 12(4): 405-12.

32. Stevens, L.A., Coresh, J., Greene, T. and Levey, A.S. (2006). "Assessing kidney function-measured and estimated glomerular filtration rate." N. Engl. J. Med. 354: 2473-83.

33. Stratta, P., Canavese, C., Messuerotti, A., Fenoglio, I. and Fubini, B. (2001). "Silica and renal diseases: no longer a problem in the 21 st century?" J. Nephrol. 14(4): 228-47. 
34. Yamano,Y., Kagawa, J., Hanaoka, T., Takahashi, T., Kasai, H., Tsugane, S. and Watanabe, S. (1995). "Oxidative DNA damage induced by silica in vivo." Environ. Res. 69: 102-7.

35. WHO, (2006). "Obesity and overweight." Geneva: World Health Organization. Factsheet 311.

36. Zhang, J.W., Lv, G.C., Yao, J.M. and Hong, X.P. (2010). "Assessment of serum antioxidant status in patients with silicosis." J. Int. Med. Res. 38(3): 884-9.

37. Zhang, Z., Shen, H.M., Zhang, Q.F. et al., (1999). "Critical role of GSH in silica-induced oxidative stress, cytotoxicity, and genotoxicity in alveolar macrophages.” Am. J. Physiol. 277: L743-8. 